\title{
MICRON-SIZED POLYMERIC PARTICLES FROM CASHEW NUT SHELL LIQUID: INFLUENCE OF REACTION PARAMETERS ON PARTICLE SIZE
}

\author{
YMM Makame, W Johnson and LL Mkayula \\ Chemistry Department, University of Dar es Salaam \\ P. O. Box 35061 Dar es Salaam, Tanzania.
}

\begin{abstract}
Cashew nut shell liquid based polymer latexes of narrow size distribution and average particle sizes ranging from 0.1 to $0.4 \mu \mathrm{m}$ were prepared by base catalysed emulsion polycondensation of cashew nut shell liquid of 820 centipoises viscosity with formaldehyde. The influence of emulsifier concentration, sodium hydroxide concentration and stirring rate on average particle size and size distribution was studied for the given geometry of the reactor and the stirrer. It was found that these variables have a significant effect on the particle size and particle size distribution and that average particles size can be predicted and controlled by the choice of these experimental variables. Interrelationships between these variables appear to be complex, however the apparent monotonic variation of average particle size with emulsifier concentration makes emulsifier concentration the most convenient parameter of controlling particle size. The influence of the type of mixers and various reaction kettle designs on the polymer particles' sizes should be studied.
\end{abstract}

\section{INTRODUCTION}

Cashew (Anacardium occidentale L.) Nut Shell Liquid (CNSL) is a natural source for unsaturated long-chain phenols. Obtained as a byproduct of the cashew industry, this cheaply available material holds considerable promise as a renewable natural monomer for polymer production. CNSL is classified as<smiles>[R]c1cccc(O)c1</smiles>

1<smiles>[R]c1cc(O)cc(O)c1</smiles>

2<smiles>[R]c1cc(O)c(C)c(O)c1</smiles>

3 natural or technical depending on the method of extraction from cashew nut shell; natural CNSL and technical CNSL refer to CNSL extracted by solvent and by thermal treatment, respectively. The major components of natural CNSL are cardanol (1), cardol (2), 2- methylcardol (3) and anacardic acid (4) (Tyman 1975).$$
1
$$

In general, CNSL based polymeric materials have been prepared by condensation reaction of CNSL with active methylenes such as formaldehyde, paraformaldehyde and hexamethylenetetramine, or by addition polymerization through the unsaturation in the side chain. (Gedam and Sampathkumaran 1986) and (Anand 1978) have reviewed the various applications of cashew nut shell liquid, and of CNSL based<smiles>[R]c1cccc(O)c1C(=O)O</smiles>

polymers, respectively. Recently Mwaikambo and Ansell (2001) studied thermo-properties and cure characteristics of CNSL-based resins. Bisanda and Ansell (1992) reported the preparation of CNSLformaldehyde resin for use in sisal fiber reinforced composites. Bhunia et al. (1998) reported the synthesis of polyurethane from cashew nut shell liquid. 
Small-size spherical polymer particles find a wide variety of applications including packing material for chromatographic separation, support materials for biochemicals and heterogeneous catalysts. In particular micron-size monodisperse spherical polymer particles can be applied in biomedical and microelectronic fields. The level of success in each of these applications is dependent upon the particle size and its distribution, the morphology and surface characteristics of the particles among other factors (Lok and Ober 1985, Okobu et al. 1989). Polymer particles of predetermined particle size range can be obtained by employing suitable polymerization techniques with careful control of reaction conditions and parameters. Emulsion-, suspension- and dispersion- polymerizations have been used to that effect (Arshady 1992). The average size and size-distribution of the particles as well as the kinetics of the polymerization process depend on a number of parameters such as emulsifier and stabilizer concentrations, solvency of the dispersion medium, stirring rate and type of mixers and temperature. Ilomo et al. (2004) prepared micron-sized polymer particles (MSPP) from CNSL through emulsion polycondensation method. The average sizes of the MSPP that ranged from 0.1 to 4.4 $\mu \mathrm{m}$ were found to be influenced by emulsifier concentration, catalyst $(\mathrm{NaOH})$ concentration and agitation speed. The present work intends to complement Ilomo's results by systematically analyzing and describing the effects of emulsifier concentration, catalyst concentration and stirring speed on the average particle sizes of MSPP using more advanced TEM and CRYO-SEM techniques.

\section{EXPERIMENTAL}

\section{Reagents}

Technical CNSL was supplied by TANITA Company Limited based in Dar es Salaam, Tanzania. $\mathrm{NaOH}$ pellets, formalin $(37-41 \%$ $\mathrm{w} / \mathrm{v}$ aq. solution) and Sodium Lauryl Sulphate (NaLS) were A.R. grade supplied by Unilab. Other reagents and solvents were
A.R. grade supplied by BDH Chemicals. Distilled water was used in all preparations. Preparation of Micron-Sized Polymer
Particles (MSSP)

Latexes of micron-sized polymer particles were prepared by base $(\mathrm{NaOH})$ catalysed reaction of CNSL with formalin at $60^{\circ} \mathrm{C}$, with sodium lauryl sulphate (NaLS) as the emulsifier using the recipes presented in Table 1. The procedure outlined by Ilomo (2001) was adopted. Two-litres' capacity cylindrical batch reactor and propeller-headed stainless steel stirrer were used and polymerization time was 6 hours. The CNSL based MSPP was obtained as yellowish brown latexes, which on filtering, washing and drying produced brownish powdered solid materials.

Investigating the effects of experimental variables on average particles sizes

The effects of emulsifier (NaLS) and catalyst $(\mathrm{NaOH})$ concentrations in the reaction mixtures, and that of reaction mixture's speed of agitation on size of the resulting polymer particles were investigated. The polymerization recipes were prepared in such a way that in a set of four or five experiments only the values of one variable were varied while the values of the other two variables were kept constant. The first set of experiments was performed with $[\mathrm{NaLS}]$ in the recipes gradually increased from $2,3,4$, 6 to $8 \mathrm{~g} / \mathrm{dm}^{3}-\mathrm{H}_{2} \mathrm{O}$ (Fig. 3) while $[\mathrm{NaOH}]$ and stirring rate were kept constant at 1.2 $\mathrm{g} / \mathrm{dm}^{3}-\mathrm{H}_{2} \mathrm{O}$ and $600 \mathrm{rpm}$ respectively. The second set was carried out using aqueous $\mathrm{NaOH}$ at concentrations of $0.6,0.8,1.0$ and $1.2 \mathrm{~g} / \mathrm{dm}^{3}-\mathrm{H}_{2} \mathrm{O}$ (Fig. 2) while [NaLS] and stirring rate were kept constant at $4.0 \mathrm{~g} / \mathrm{dm}^{3}$ $\mathrm{H}_{2} \mathrm{O}$ and $600 \mathrm{rpm}$, respectively. To investigate the effect of agitation speed on resulting polymer particles sizes, the stirring rate, in a set of five recipes, was varied from $200,400,600,800$ to $1000 \mathrm{rpm}$ while $[\mathrm{NaLS}]$ and $[\mathrm{NaOH}]$ were kept constant at $4.0 \mathrm{~g} / \mathrm{dm}^{3}-\mathrm{H}_{2} \mathrm{O}$ and $1.2 \mathrm{~g} / \mathrm{dm}^{3}-\mathrm{H}_{2} \mathrm{O}$. 
Determination of polymer particles' sizes The average particles sizes and sizes distributions of MSPP were determined by Transmission Electron Microscopy (TEM JOEL 901) and CRYO-SEM Scanning Electron Microscopy.

\section{RESULTS AND DISCUSSION}

Preparation of micron sized polymer particles (MSPP) from CNSL

The emulsion polycondensation of CNSL with formaldehyde produced clean, stable latexes except in samples prepared with low emulsifier concentrations at low agitation rates, in which cases, limited coagulation was evident. It was apparent that turbulentfree uniform distribution of the stirring force throughout the suspension mixture was essential to minimize the chance of inadvertent particle coagulation and hence production of polymer particles with correspondingly narrow size distribution. The sizes of the individual particles ranged from 0.1 to $1 \mu \mathrm{m}$. The three-dimensional Cryo-SEM picture (Fig. 1) indicates that the MSPP were spherical in shape. The spherical shape could be attributed to the stability of the suspension system.

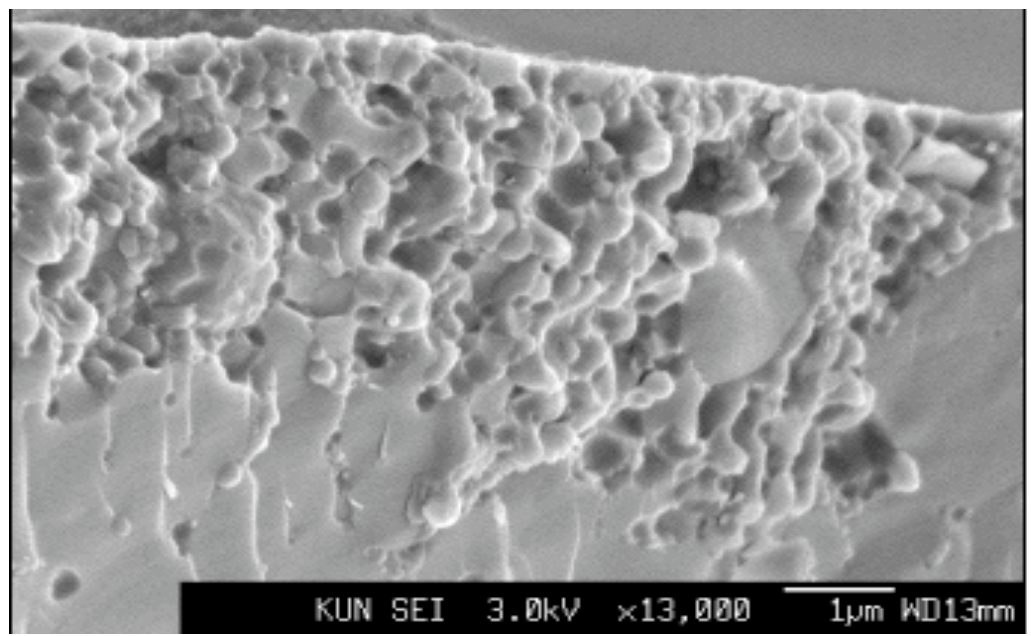

Figure 1: Cryo-SEM Micrograph for MSPP - Recipe no. 1

The average sizes of the MSPP obtained from the different recipes were determined and shown in table 1 . The average particle sizes are reproducible in that different recipes of nearly similar composition produced particles of effectively same average size. For instance replicate recipes no. 3 and no. 8 gave average particle sizes of $0.274 \mu \mathrm{m}$, and $0.269 \mu \mathrm{m}$ respectively.

Effect $\mathrm{NaOH}$ concentration on the average particle sizes

The effect of $\mathrm{NaOH}$ concentration on the average size of the resulting polymer particles was purposely studied since $\mathrm{NaOH}$ is normally used as catalyst in CNSLformaldehyde condensation reactions whereby the alkali activates the phenolic groups of CNSL components molecules. $\mathrm{NaOH}$ concentration was varied within the levels commonly used in phenolformaldehyde condensation polymerization. As seen in Fig. 2, an increase in sodium hydroxide concentration from 0.6 to 1.2 $\mathrm{g} / \mathrm{dm}^{3}$ has some effect on the size of the polymer particles formed; by increasing $\mathrm{NaOH}$ concentration from 0.6 to $1.0 \mathrm{~g} / \mathrm{dm}^{3}$ the average particle size decreased from 0.28 
Makame et al. - Micron-sized polymeric particles from cashew nut shell liquid ...

to $0.21 \mu \mathrm{m}$. When the concentration of $\mathrm{NaOH}$ was increased further to $1.2 \mathrm{~g} / \mathrm{dm}^{3}$ while keeping constant the values of the other variables, the extent of coagulation in the latex was found to have intensified; this gave rise to the increase in particle size to $0.21 \mu \mathrm{m}$ and correspondingly to the broadening of particle size distribution. Generally a slight increase in particle size with increasing $\mathrm{NaOH}$ concentration was expected due to the effect of $\mathrm{NaOH}$ on both the ionic strength and the viscosity of the aqueous dispersion medium. According to Goodwin et al. (1978) ionic strength of the aqueous phase influences the course of particle nucleation process and plays important role in determining the size of the polymer particles formed. The presence of $\mathrm{NaOH}$ is also expected to counteract the influence of emulsifier in the system; while the emulsifier functions by stabilizing polymer latexes, strong electrolytes, like $\mathrm{NaOH}$, tend to destabilize such polymer colloidal systems.

Table 1: Reaction compositions, stirring rates and the average particle sizes

\begin{tabular}{|c|c|c|c|c|c|c|c|}
\hline \multirow[b]{2}{*}{ Exp. } & \multicolumn{5}{|c|}{ Amounts of reagents } & \multirow{2}{*}{$\begin{array}{c}\text { Stirring } \\
\text { Speed } \\
\text { (rpm) } \\
\end{array}$} & \multirow{2}{*}{$\begin{array}{c}\text { Average Particle } \\
\text { Size } \\
(\mu \mathrm{m})\end{array}$} \\
\hline & $\begin{array}{c}\text { CNSL } \\
\pm 0.1 \mathrm{~g} \\
\end{array}$ & $\begin{array}{c}\text { Formalin } \\
\pm \mathbf{0 . 1} \mathbf{g} \\
\end{array}$ & $\begin{array}{r}\mathrm{H}_{2} \mathrm{O} \\
\pm 1 \mathrm{~g} \\
\end{array}$ & $\begin{array}{c}\mathrm{NaOH} \\
\pm 0.01 \mathrm{~g} \\
\end{array}$ & $\begin{array}{c}\text { NaLS } \\
\pm 0.01 \mathrm{~g} \\
\end{array}$ & & \\
\hline 1 & 58.9 & 32.5 & 1000 & 1.21 & 2.02 & 600 & 0.389 \\
\hline 2 & 59.0 & 32.2 & 1000 & 1.23 & 3.06 & 600 & 0.342 \\
\hline 3 & 59.1 & 32.1 & 1000 & 1.22 & 4.02 & 600 & 0.274 \\
\hline 4 & 59.7 & 32.2 & 1001 & 1.22 & 6.06 & 600 & 0.237 \\
\hline 5 & 58.5 & 32.1 & 1000 & 1.27 & 8.08 & 600 & 0.145 \\
\hline 6 & 58.7 & 32.3 & 1001 & 1.26 & 4.04 & 200 & 0.324 \\
\hline 7 & 58.8 & 32.0 & 1000 & 1.27 & 4.04 & 400 & 0.303 \\
\hline 8 & 58.2 & 32.6 & 1000 & 1.21 & 4.05 & 600 & 0.269 \\
\hline 9 & 58.8 & 32.4 & 1001 & 1.26 & 4.04 & 800 & 0.236 \\
\hline 10 & 58.5 & 32.6 & 1000 & 1.23 & 4.08 & 1000 & 0.314 \\
\hline 11 & 58.7 & 32.1 & 1000 & 0.61 & 4.05 & 600 & 0.284 \\
\hline 12 & 58.7 & 32.3 & 1001 & 0.80 & 4.02 & 600 & 0.234 \\
\hline 13 & 58.7 & 32.4 & 1001 & 1.00 & 4.06 & 600 & 0.209 \\
\hline
\end{tabular}

Effect of the emulsifier (NaLS) concentration on the average particle sizes As seen in Fig. 3, by increasing the emulsifier concentration from 2 to $8 \mathrm{~g} / \mathrm{dm}^{3}$, the average particle sizes became increasingly smaller. This implies that emulsifier concentration, in the range covered in this work, correlated inversely with the average particle size. This is not a surprising observation since the more the amount of emulsifier the larger the surface area that can be stabilized and surface area per unit volume of a spherical particle increases with a decrease in size. Low levels of emulsifier concentration were found to broaden size distribution of the resulting polymer particles. The linear graph (Fig. 4) with regression value of 0.982 , suggests more or less linearity in the trend. In similar study by Ilomo (2001) an increase in emulsifier concentration coupled with a decrease in $\mathrm{NaOH}$ concentration was found to drastically reduce the mean size of the resulting polymer particles. The present results that are based on more advanced TEM and CRYO-SEM techniques appear to complement Ilomo's results. 


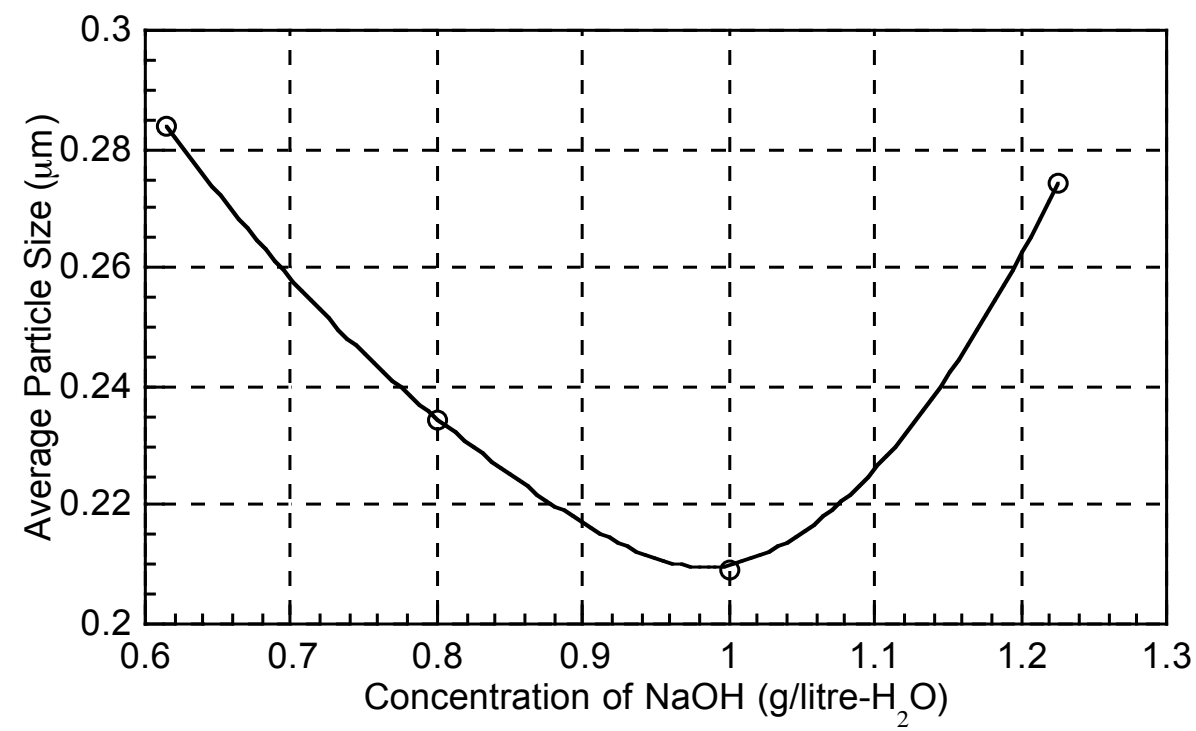

Figure 2: The effect of $\mathrm{NaOH}$ concentration on the average particle size (stirring rate $=600$ rpm, $[\mathrm{NaLS}]=4 \mathrm{~g} /$ litre)

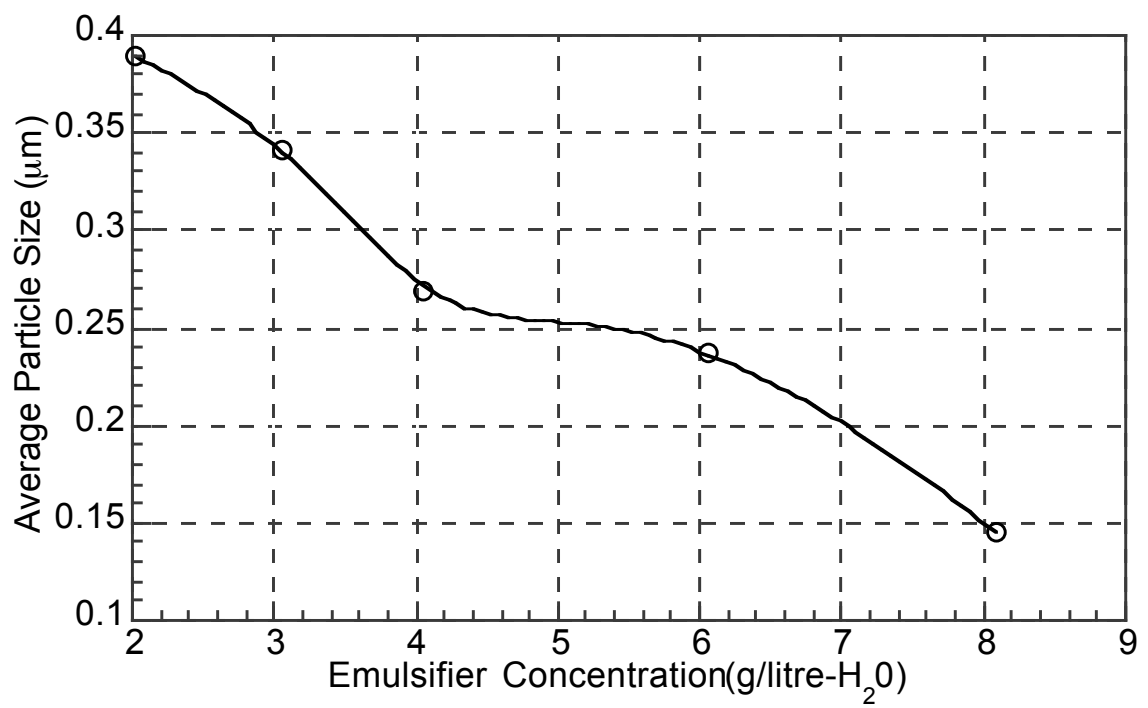

Figure 3: The effect of emulsifier concentration on the average particle size (smooth curve) (stirring rate $=600 \mathrm{rpm},[\mathrm{NaOH}]=1.2 \mathrm{~g} /$ litre) 


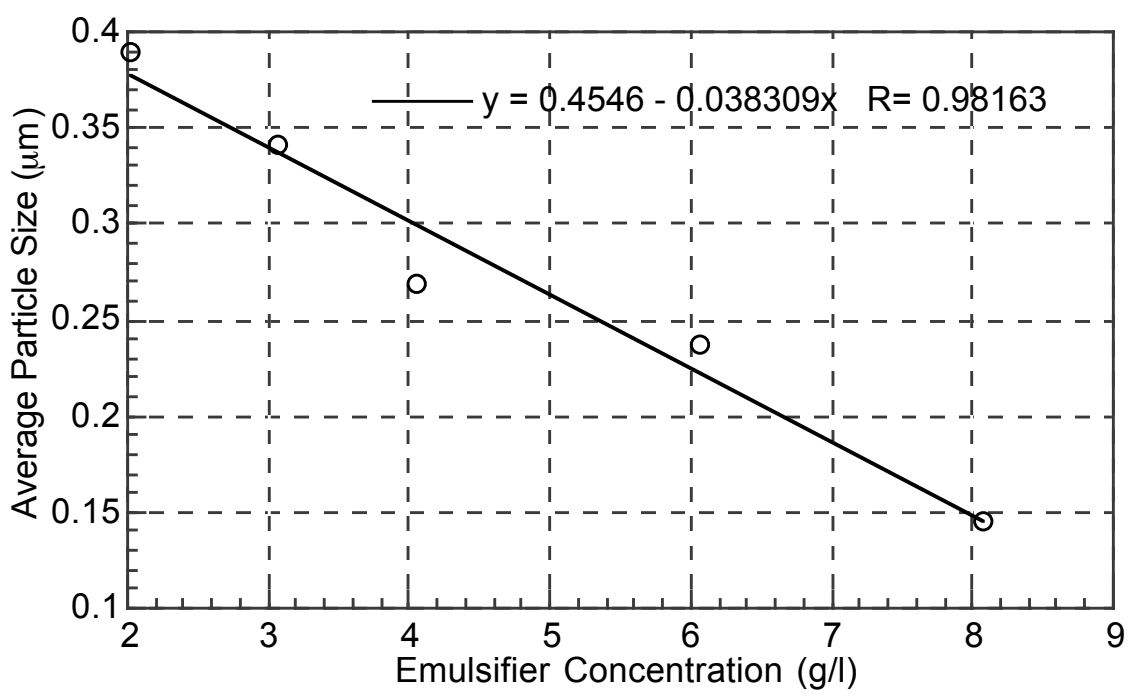

Figure 4: The effect of emulsifier concentration on the average particle size (linear graph) (stirring rate $=600 \mathrm{rpm},[\mathrm{NaOH}]=1.2 \mathrm{~g} /$ litre)

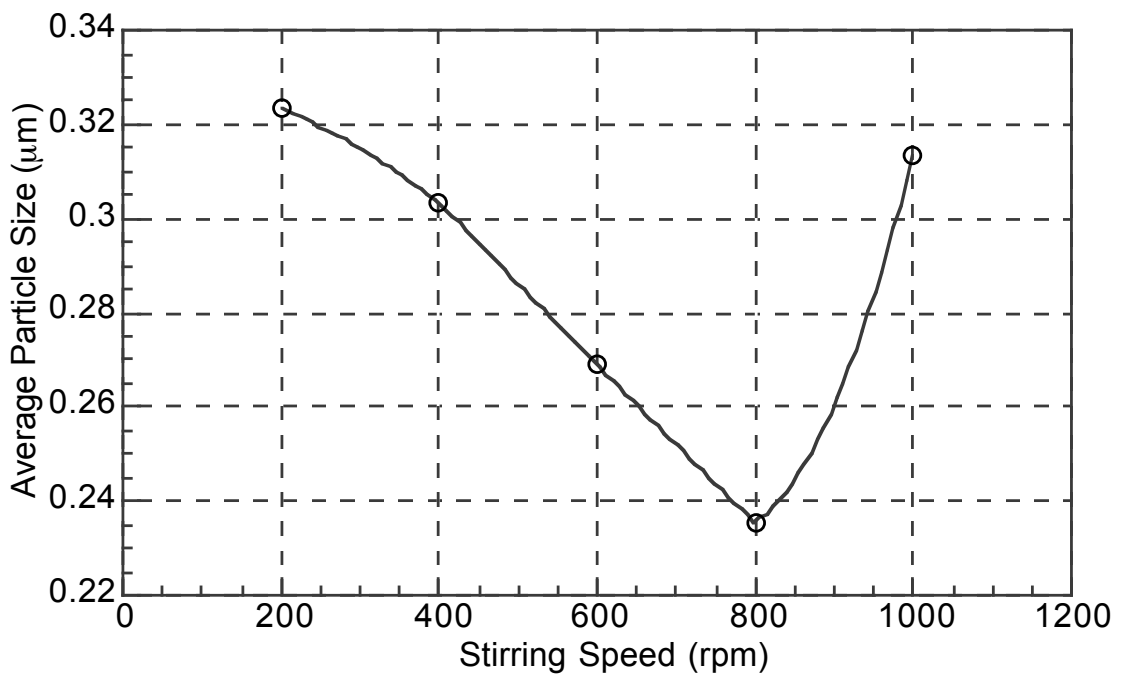

Figure 5: The effect of stirring rate on the average particle size (smooth curve) $[\mathrm{NaLS}]=4$ $\mathrm{g} /$ litre- $\mathrm{H}_{2} \mathrm{O} .[\mathrm{NaOH}]=1.2 \mathrm{~g} /$ litre- $\mathrm{H}_{2} \mathrm{O}$.

Effect of stirring rate on the average particle size

The stirring rate was varied step-wise, from $200,400,600,800$ to $1000 \mathrm{rpm}$. Higher agitations than $1000 \mathrm{rpm}$ caused turbulence and non-uniform mixing of reagents. The dependence of average particle size on stirring rate is provided by the graphs in figures 5 and 6 . Figure 5 shows that within the speed range 200 to $800 \mathrm{rpm}$, the average particle size decreased with increase in stirring speed. Thereafter, at higher stirring 
speed, $(>800 \mathrm{rpm})$ the increase in stirring speed resulted in an increase in the average particle size. Similar behavior was observed by Ahmed (1984) for particles formed by suspension polymerization of styrene. Generally, the average particle diameters decreased with increasing stirring speed. However, with lower levels of stabilizer the final average particle diameters first decreased with increasing speed of stirring, reached a minimum, then increased rapidly and finally leveled off. According to Ahmed (1984) the observation could be explained in terms of the surface coverage of the particles with stabilizers. Since the size of the monomer droplets is generally determined by the intensity of mixing, then higher speed would give very small initial droplets thereby increasing the total surface area of the droplets. The amount of stabilizer originally present would seem to be inadequate to protect against coalescence thus increasing the final particle size. The increase in average size with stirring rate (above $800 \mathrm{rpm}$ ) can probably be attributed to coagulation of the soft, partly polymerized particles due to the excessive stirring forces.

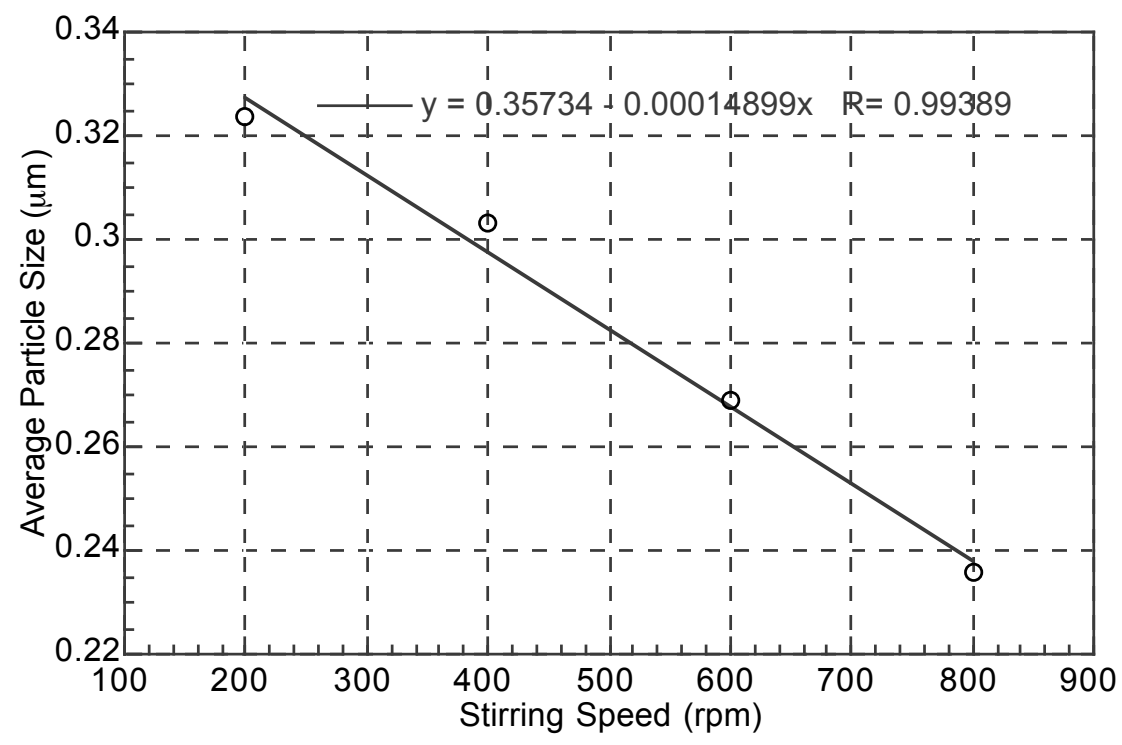

Figure 6: The effect of stirring rate on the average particle size (linear graphing) $[\mathrm{NaLS}]=4$ g/litre- $\mathrm{H}_{2} \mathrm{O} .[\mathrm{NaOH}]=1.2 \mathrm{~g} /$ litre- $\mathrm{H}_{2} \mathrm{O}$

Linear plotting the data between 200 and $800 \mathrm{rpm}$ (fig. 6) resulted into a linear regression coefficient, R-value, of 0.994. The R-value obtained suggests the existence of linear relationship between the stirring speed and the average particle size within that specified range of agitation speed under the given reaction condition. The slope of the line is expected to vary depending on the magnitude of other parameters. Since the relationship is linear then the slope can be used to predict the size of the particles for a particular recipe at a particular stirring speed. The reason for this linearity is not quite clear and is expected to be complex. Nevertheless, apparently the average size of the resulting CNSL-based MSPP can be readily controlled by varying the stirring 
speed within a limited range of stirring speed. However, over a long range of stirring speeds, the correlation between the average particle size and stirrer speed, can change with stirring speed even when other reaction parameters have been maintained constant.

Stirrer (mixer) design is among important parameters in stirring systems. Brooks (1990) discovered differences in mass median diameters and particle size distribution as mixers were periodically exchanged in suspension polymerization systems. However the effect due to stirrer design was not investigated in this study.

\section{CONCLUSION}

Emulsion polycondensation of cashew nut shell liquid (CNSL) with formaldehyde was employed successfully in preparing CNSL based Micron-sized Polymeric Particles (MSPP) of average particle size from about 0.1 to $0.4 \mu \mathrm{m}$ with narrow, well-defined particle size distribution. It is evident that subtle changes in reaction parameters such as stirring rate, and emulsifier and catalyst concentrations be used to alter the average sizes of MSPP in a systematic and wellcontrolled manner, however, monotonic variation of average particle size with emulsifier concentration makes emulsifier concentration the most convenient parameter of controlling particle size. The influence of the type of mixers and various reaction kettle designs on MSPP size should be studied.

\section{ACKNOWLEDGEMENT}

Financial support by the Norwegian Agency for International Development (NORAD) through the Head of Chemistry Department, University of Dar es Salaam is highly appreciated. We are also thankful to the members of the electron microscope unit of the Nijmegen University, the Netherlands, for Cryo-SEM micrographs.

\section{REFERENCES}

Ahmed SM 1984 Effect of agitation and the nature of protective colloid on particle size during suspension polymerization J. Disp. Sci. Technol., 5: 421-432

Anand LC 1978 CNSL based polymers and their applications Paintindia 28: 13 - 33.

Arshady R 1992 Suspension, emulsion and dispersion polymerization: A methodological survey Colloid. Polym. Sci. 270: $717-732$

Bisanda ETN and Ansell MP 1992 Sisal Fibre Reinforced Composites $J$. Material Sci, 27: 1690.

Bhunia HP, Jana RN, Basak A, Lenka S and Nando GB 1998 Synthesis of polyurethane from Cashew Nut Shell Liquid (CNSL), a renewable resource J. Polym. Sci. Part A: Polym. Chem. 36: 391 - 400.

Brooks BW 1990 Basic aspects and recent developments in suspension polymerization. Macromol. Chem., Macromol. Symp. 35/36: 121 - 140

Gedam PH and Sampathkumaran PS 1986 Cashew Nut Shell Liquid: extraction, chemistry and applications Prog. Org. Coatings, 14: 115-157.

Goodwin JW, Ottewill RH, Pelton R, Vianello, G and Yates DE, 1978 Control of particle size in the formation of polymer lattices. Brit. Polym. J. 10: 173 -180.

Ilomo OO 2001 Preparation and characterization of polymeric materials from Tanzanian cashew nut shell liquid. MSc. Thesis, University of Dar es Salaam, Tanzania.

Ilomo OO, Makame, YMM and Mkayula, LL 2004 Micron-sized polymer particles from Tanzanian cashew nut shell liquid. Part I: Preparation, functionalization with chloroacetic acid and utilization as cation exchange resin. Bull. Chem. Soc. Ethiop. 18(1): 81- 90. 
Lok KP and Ober CK 1985, Particle size control in dispersion polymerization of polystyrene. Can. J. Chem. 63: $209-216$.

Mwaikambo LY and Ansell MP (2001) Cure characteristics of alkali catalysed cashew nut shell liquid-formaldehyde resins. $J$. Materials Sci. 36: 3693 - 3698.
Okubo M, Ikegami $\mathrm{K}$ and Yamamoto, $\mathrm{Y}$ 1989 Preparation of micron-size monodisperse polymer microspheres having chloromethyl group Colloid Polym Sci. 267: 193 - 200.

Tyman JHP 1975 Long-chain phenols: V. Gas chromatographic analysis of cashew nut shell liquid (anacardium occidentale) J. Chromatogr. 111: $285-292$. 